\title{
Design of 4x4 bit Vedic Multiplier using EDA Tool
}

\author{
Pushpalata Verma \\ Assistant Professor (CSE) \\ C.C.E.M., Raipur \\ Chhattisgarh, India
}

\begin{abstract}
The need of high speed multiplier is increasing as the need of high speed processors are increasing. A Multiplier is one of the key hardware blocks in most fast processing system which is not only a high delay block but also a major source of power dissipation. A conventional processor requires substantially more hardware resources and processing time in the multiplication operation, rather than addition and subtraction. This paper presents a high speed $4 \times 4$ bit Vedic Multiplier (VM) based on Vertically \& Crosswise method of Vedic mathematics, a general multiplication formulae equally applicable to all cases of multiplication. It is based on generating all partial products and their sum in one step. The coding is done in VHDL (Very High Speed Integrated Circuit Hardware Descriptive Language) while the synthesis and simulation is done using EDA (Electronic Design Automation) tool - XilinxISE12.1i. The combinational path delay of $4 \times 4$ bit Vedic multiplier obtained after synthesis is compared with normal multipliers and found that the proposed Vedic multiplier circuit seems to have better performance in terms of speed.
\end{abstract}

\section{General Terms}

Vedic Multiplier (VM)

\section{Keywords}

Vedic Multiplication, Urdhva Tiryakbhyam Sutra, Ripple Carry Adder.

\section{INTRODUCTION}

Multipliers are extensively used in Microprocessors, DSP and Communication applications. For higher order multiplications, a huge number of adders are to be used to perform the partial product addition. The need of low power and high speed Multiplier is increasing as the need of high speed processors are increasing. The Vedic multiplication technique is based on 16 Vedic sutras or aphorisms, which are actually word formulae describing natural ways of solving a whole range of mathematical problems [1]. The mathematical operations using, Vedic Method are very fast and requires less hardware, this can be used to improve the computational speed of processors. This paper describes the design and implementation of $4 \times 4$ bit Vedic multiplier based on UrdhvaTiryakbhyam sutra (Vertically and Crosswise technique) of Vedic Mathematics using EDA (Electronic Design Automation) tool. The paper is organized as follows. Section 2 describes the basic methodology of Vedic multiplication technique. Section 3 describes the hardware architecture of $2 \times 2$ and $4 \times 4$ bits Vedic Multiplier (VM) based on Vedic multiplication. Section 4 illustrates the implementation and result of Vedic multiplier module so obtained while Section 5 comprises of Conclusion. Section 6 is the Acknowledgment whereas Section 7 comprises of References.

\section{ANCIENT VEDIC METHODS}

The use of Vedic mathematics lies in the fact that it reduces the typical calculations in conventional mathematics to very simple ones. This is so because the Vedic formulae are claimed to be based on the natural principles on which the human mind works. Vedic Mathematics is a methodology of arithmetic rules that allow more efficient speed implementation. It also provides some effective algorithms which can be applied to various branches of engineering such as computing.

\subsection{Vertically \& Crosswise Technique}

The proposed Vedic multiplier is based on the "Urdhva Tiryakbhyam" sutra (algorithm). These Sutras have been traditionally used for the multiplication of two numbers in the decimal number system. In this work, we apply the same ideas to the binary number system to make the proposed algorithm compatible with the digital hardware. It is a general multiplication formula applicable to all cases of multiplication. It literally means "Vertically and crosswise". It is based on a novel concept through which the generation of all partial products can be done with the concurrent addition of these partial products. The algorithm can be generalized for $\mathrm{n} \times \mathrm{n}$ bit number. Since the partial products and their sums are calculated in parallel, the multiplier is independent of the clock frequency of the processor. The Multiplier based on this sutra has the advantage that as the number of bits increases, gate delay and area increases very slowly as compared to other conventional multipliers.

To illustrate this scheme, let us consider the multiplication of two decimal numbers $252 * 846$ by Urdhva Tiryakbhyam method as shown in Figure 1. The digits on the both sides of the line (say, 2 and 6$)$ are multiplied $(6 \times 2=12)$ and added with the carry from the previous step (initially, carry $=0$ ). This generates one of the bits of the result (i.e. 2) and a carry (i.e. $1)$. This carry (1) is added in the next step and hence the process goes on. If more than one line are there in one step, all the results are added to the previous carry. In each step, Least Significant Bit (LSB) acts as the result bit and all other bits act as carry for the next step. Initially the carry is taken to be zero. 


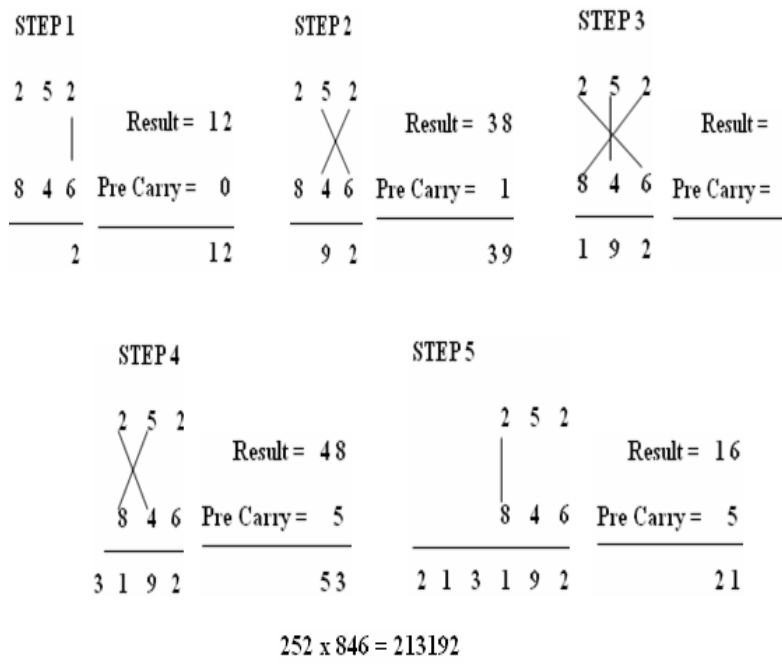

Figure 1: Multiplication of two decimal numbers $252 * 846$

\section{PROPOSED VEDIC ARCHITECTURE}

The hardware architecture of $2 \times 2$ and $4 \times 4$ bit Vedic multiplier (VM) modules are displayed in the below sections. Here, "Urdhva-Tiryakbhyam" (Vertically and Crosswise) sutra is used to propose such an architecture for the multiplication of two binary numbers. The beauty of Vedic multiplier is that here partial product generation and additions are done concurrently. Hence, it is well adapted to parallel processing. The feature makes it more attractive for binary multiplications. This in turn reduces delay.

\subsection{Vedic Multiplier for $2 \times 2$ bit}

The method is explained below for two, 2 bit numbers $A$ and $B$ where $A=a 1 a 0$ and $B=b 1 b 0$ as shown in Figure 2. Firstly, the Least Significant Bits are multiplied which gives the Least Significant Bit (LSB) of the final product (vertical). Then, the LSB of the multiplicand is multiplied with the next higher bit of the multiplier and added with, the product of LSB of multiplier and next higher bit of the multiplicand (crosswise). The sum gives second bit of the final product and the carry is added with the partial product obtained by multiplying the most significant bits to give the sum and carry. The sum is the third corresponding bit and carry becomes the fourth bit of the final product.

$$
\begin{aligned}
& s 0=a 0 b 0 \\
& c 1 s 1=a 1 b 0+a 0 b 1 ; \\
& c 2 s 2=c 1+a 1 b 1
\end{aligned}
$$

The final result will be $c 2 s 2 s 1 s 0$. This multiplication method is applicable for all the cases. The $2 \times 2$ bit Vedic multiplier (VM) module is implemented using four input AND gates \& two half-adders which is displayed in its block diagram in Figure 3.

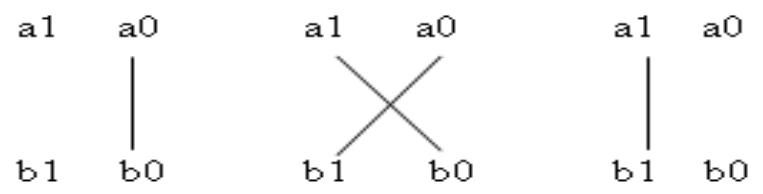

Figure 2: The Vedic Multiplication Method for two 2-bit binary numbers

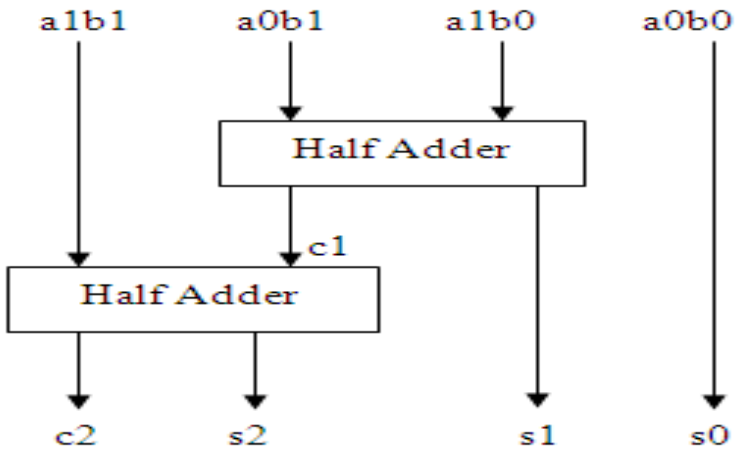

Figure 3: Block Diagram of 2x2 bit Vedic Multiplier (VM)

The same method can be extended for higher no. of input bits (say 4). But a little modification is required as discussed in section 3.2. This section illustrates the implementation of $4 \times 4$ bit $\mathrm{VM}$ which uses $2 \times 2$ bit $\mathrm{VM}$ as a basic module.

\subsection{Vedic Multiplier for $4 \times 4$ bit}

Divide the no. of bits in the inputs equally in two parts. Let's analyze $4 \times 4$ bit multiplication, say multiplicand $\mathrm{A}=\mathrm{A} 3 \mathrm{~A} 2 \mathrm{~A} 1 \mathrm{~A} 0$ and multiplier $\mathrm{B}=\mathrm{B} 3 \mathrm{~B} 2 \mathrm{~B} 1 \mathrm{~B} 0$. Following are the output line for the multiplication result, S7S6S5S4S3S2S1S0. Let's divide A and B into two parts, say "A3 A2" \& "A1 A0" for A and "B3 B2" \& "B1B0" for B. Using the fundamental of Vedic multiplication, taking two bit at a time and using 2 bit multiplier block, we can have the following structure for $4 \times 4$ bit multiplication as shown in Figure 4.

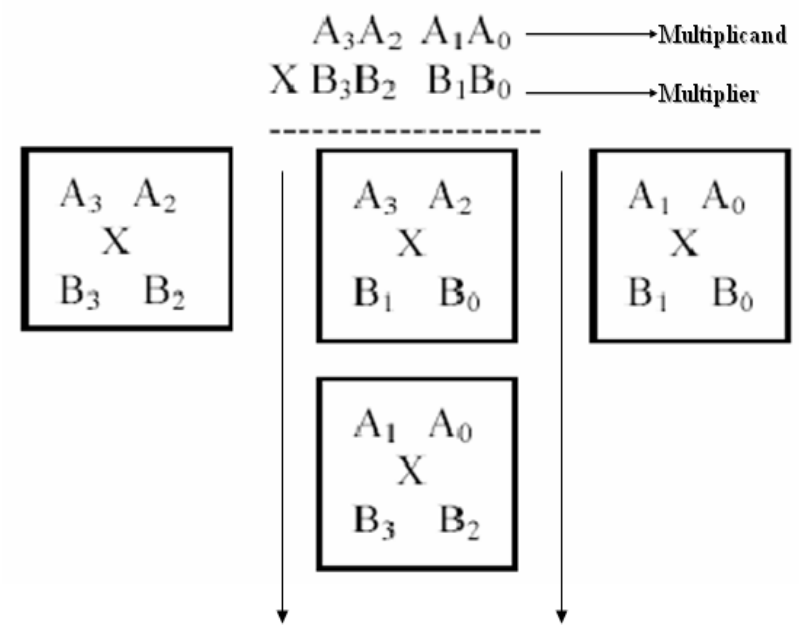

Figure 4: Structure for $4 \times 4$ bit Multiplication

Each block as shown above is $2 \times 2$ bit multiplier. First $2 \times 2$ multiplier inputs are "A1 A0" and "B1 B0". The last block is $2 \times 2$ bit multiplier with inputs "A3 A2" and "B3 B2". The middle one shows two, $2 \times 2$ bit multiplier with inputs "A3A2" \& "B1B0" and "A1A0" \& "B3B2". So the final result of multiplication, which is of 8 bit, "S7S6S5S4S3S2 S1S0".

To understand the concept, the block diagram of $4 \times 4$ bit Vedic multiplier is shown in Figure 5. To get final product S7S6S5S4S3S2S1S0 four, 2-bit Vedic multiplier (Figure 3) 
and three 4-bit Ripple Carry (RC) Adders are required. In this proposal, the first 4-bit RC Adder is used to add two 4-bit operands obtained from cross multiplication of the two middle $2 \times 2$ bit multiplier modules. The second 4-bit RC Adder is used to add two 4-bit operands, i.e. concatenated 4-bit ("00" $\&$ most significant two output bits of right hand most of $2 \times 2$ multiplier module as shown in Figure 5) and one 4-bit operand we get as the output sum of first RC Adder. Its carry "ca1" is forwarded to third RC Adder. Now the third 4-bit RC Adder is used to add two 4-bit operands, i.e. concatenated 4 bit (carry ca1, "0" \& most significant two output sum bits of $2^{\text {nd }}$ RC Adder as shown in Figure 5) and one 4-bit operand we get as the output sum of left hand most of $2 \times 2$ multiplier module. Early literature speaks about Vedic multipliers based on array multiplier structures. The arrangement of Ripple Carry Adder as shown in Figure 6 helps us to reduce delay.

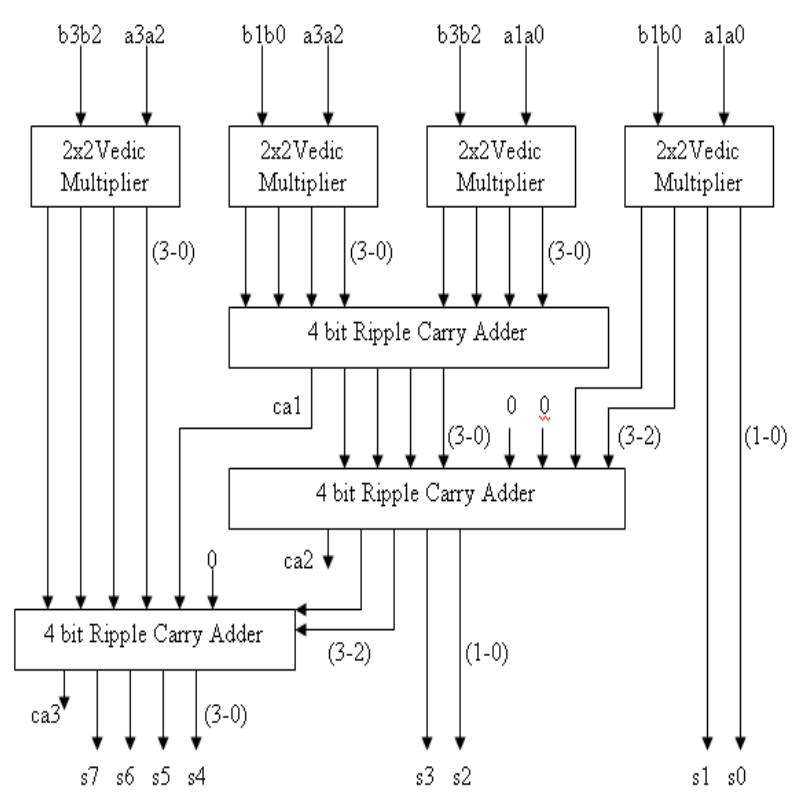

Figure 5: Block Diagram of 4x4 bit Vedic Multiplier (VM)

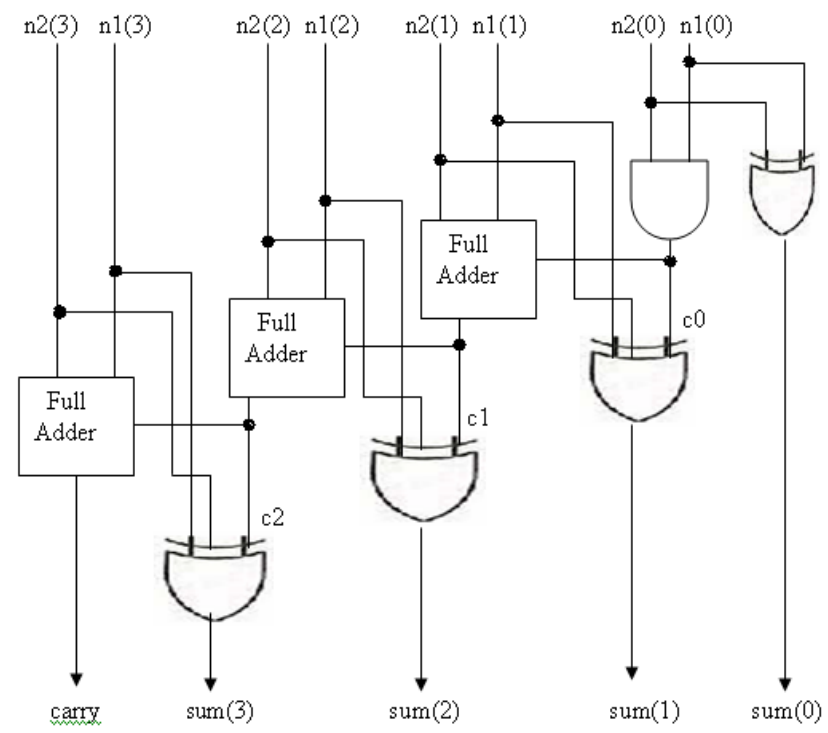

Figure 6: Circuit Diagram of 4 bit Ripple Carry Adder

\section{IMPLEMENTATION \& RESULTS}

In this work, $4 \times 4$ bit VM (Vedic multiplier) using "Urdhva Tiryakbhyam" Sutra is implemented in VHDL (Very High Speed Integrated Circuit Hardware Descriptive Language). Logic synthesis and simulation was done using EDA (Electronic Design Automation) tool in XilinxISE12.1i Project Navigator and ISim simulator integrated in the Xilinx package respectively. Table 1 displays the comparison of synthesis results of the proposed Vedic multiplier with the Conventional multipliers in terms of time delay (in nanoseconds). The combinational path delay obtained for the proposed $4 \times 4$ bit Vedic multiplier is $13.102 \mathrm{~ns}$ whereas the results of $4 \times 4$ bit Array and Booth multipliers have been taken from Umesh Akare et al [16].The performance is evaluated on the Xilinx device family Spartan3, package tq144 and speed grade -5 .

Table 1 Comparison of Multipliers (in nanosecond)

\begin{tabular}{|c|c|c|c|}
\hline $\begin{array}{c}\text { Device: } \\
\text { Spartan } \\
\text { xc3s50a- } \\
\text { 5tq144 }\end{array}$ & $\begin{array}{c}\text { Array } \\
\text { Multiplier }\end{array}$ & $\begin{array}{c}\text { Booth } \\
\text { Multiplier }\end{array}$ & $\begin{array}{c}\text { Vedic } \\
\text { Multiplier }\end{array}$ \\
\hline 4x4 bit VM & $32.001 \mathrm{~ns}$ & $16.276 \mathrm{~ns}$ & $13.102 \mathrm{~ns}$ \\
\hline
\end{tabular}

The RTL (Register Transfer Level) schematic of the $4 \times 4$ bit Vedic multiplier comprises of four $2 \times 2$ bit Vedic multiplier (vedic_multi_struct) v1, v2, v3, v4 and three 4-bit Ripple Carry Adder (rc_adder) v5, v6, v7 as shown in Figure 7 while the simulation results obtained are shown in Figure 8 for verification. In behavioral simulation we have tested for input bits: - "0100" (in decimal number system 4) and "0100" (decimal number system 4) as inputs and we get output as "00001000" (decimal number system 8). The inputs of 4-bits are decomposed into the input of 2-bits. The input for MSB (Most Significant Bit) \& LSB (Least Significant Bit) of multiplicand are $\mathrm{kh}=01$ and $\mathrm{kl}=00$, while the input for MSB (Most Significant Bit) \& LSB (Least Significant Bit) of multiplier are $\mathrm{lh}=00$ and $1 \mathrm{l}=01$. Finally, the output $1=00010000$, indicates the final 8-bit result. However the rest signals indicate the intermediate results like partial products (sum \& carry).

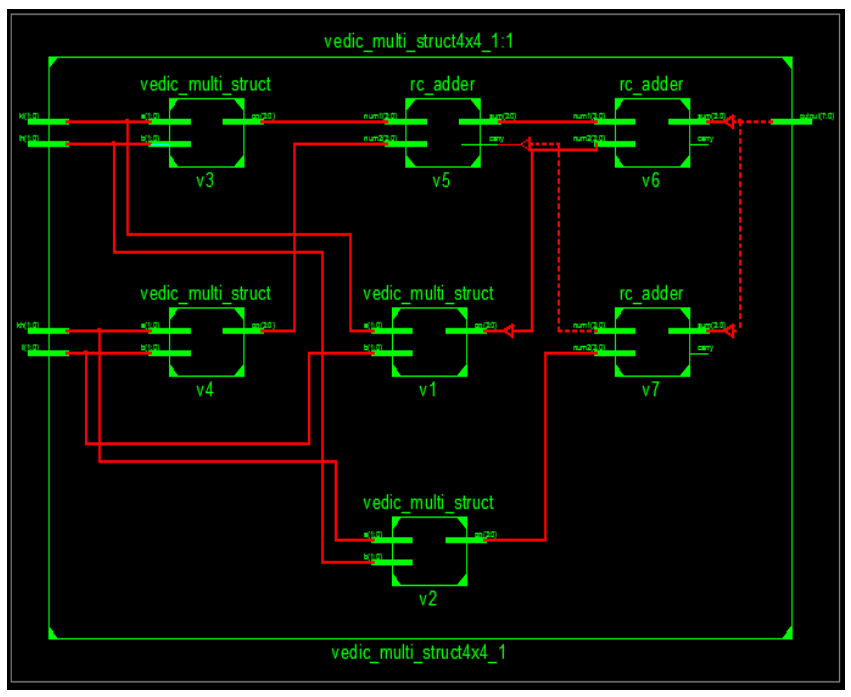

Figure 7: RTL schematic of $4 \times 4$ bit Vedic Multiplier 

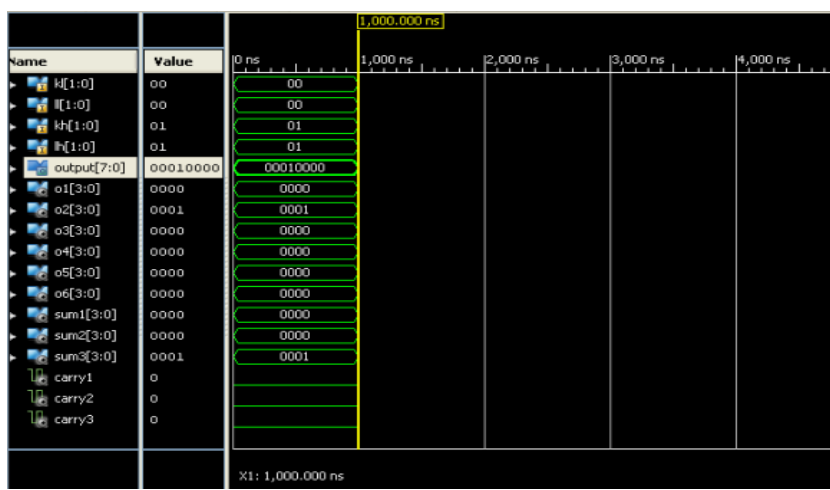

Figure 8: Simulation Result of $4 \times 4$ bit Vedic Multiplier

\section{CONCLUSION}

This paper presents a new method of multiplication "Urdhva Tiryakbhyam" Sutra based on Vedic Mathematics. The design of the proposed $4 \times 4$ bit Vedic multiplier is implemented on Spartan xc3s50a-5-tq144 device. The computational path delay of the Vedic multiplier is found to be 13.102 ns. Hence it can be concluded that the performance of the proposed $4 \mathrm{x} 4$ bit Vedic multiplier seems to be highly efficient in terms of speed when compared to Conventional multipliers. Reducing the time delay is very essential requirement for many applications and Vedic Multiplication technique is very much suitable for this purpose. The idea proposed here may set path for future research in this direction.

\section{ACKNOWLEDGMENTS}

I acknowledge with gratitude and humanity my indebtedness to Dr. K.K. Mehta, Professor and Head of Computer Science \& Engineering Department, S.S.C.E.T., Bhilai, Chhattisgarh, India, under whose guidance and support I had the privilege to complete this work.

\section{REFERENCES}

[1] Jagadguru Swami Sri Bharati Krisna Tirthaji Maharaja, "Vedic Mathematics or Sixteen Simple Mathematical Formulae from the Veda, Delhi (1965)", Motilal Banarsidas, Varanasi, India, 1986.

[2] M. Morris Mano, "Computer System Architecture", $3^{\text {rd }}$ edition, Prientice-Hall, New Jersey, USA, 1993, pp. 346-348.

[3] H. Thapliyal and H.R Arbania. "A Time-Area-Power Efficient Multiplier and Square Architecture Based On Ancient Indian Vedic Mathematics", Proceedings of the 2004, International Conference on VLSI (VLSI'04), Las Vegas, Nevada, June 2004, pp. 434-9.

[4] P. D. Chidgupkar and M. T. Karad, "The Implementation of Vedic Algorithms in Digital Signal Processing”, Global J. of Engg. Edu, Vol.8, No.2, 2004 UICEE Published in Australia.

[5] Thapliyal H. and Srinivas M.B, "High Speed Efficient NxN Bit Parallel Hierarchical Overlay Multiplier Architecture Based on Ancient Indian Vedic Mathematics", Transactions on Engineering, Computing and Technology, 2004, Vol.2.

[6] Shamim Akhter, "VHDL Implementation of Fast NXN Multiplier Based On Vedic Mathematics". Jaypee Institute of Information Technology University, Noida, 201307 UP, INDIA, 2007 IEEE.
[7] Harpreet Singh Dhillon and Abhijit Mitra, "A Reduced- Bit Multipliction Algorithm for Digital Arithmatics", International Journal of Computational and Mathematical Sciences $\quad 2.2 \quad$ @ www.waset.orgSpring2008.

[8] Honey Durga Tiwari, Ganzorig Gankhuyag, Chan Mo Kim, Yong Beom Cho, "Multiplier design based on ancient Indian Vedic Mathematician", International SoC Design Conference, 2008.

[9] Parth Mehta, Dhanashri Gawali, "Conventional versus Vedic mathematics method for Hardware implementation of a multiplier", International conference on Advance in Computing, Control, and Telecommunication Technologies, 2009.

[10] Ramalatha, M.Dayalan, K D Dharani, P Priya, and S Deoborah, "High Speed Energy Efficient ALU design using Vedic multiplication techniques", International Conference on Advances in Computational Tools for Engineering Applications, 2009. ACTEA '09.pp. 600-3, Jul 15-17, 2009.

[11] Sumita Vaidya and Deepak Dandekar, "Delay-Power Performance comparison of Multipliers in VLSI Circuit Design”, International Journal of Computer Networks \& Communications (IJCNC), Vol.2, No.4, July 2010.

[12] S.S.Kerur, Prakash Narchi, Jayashree C N, Harish M Kittur and Girish V A "Implementation of Vedic Multiplier For Digital Signal Processing" International conference on VLSI communication \& instrumentation (ICVCI) 2011

[13] Asmita Haveliya, “A Novel Design for High Speed Multiplier for Digital Signal Processing Applications (Ancient Indian Vedic mathematics approach)", International Journal of Technology and Engineering System (IJTES), Vol.2, No.1, Jan-March, 2011.

[14] Prabha S., Kasliwal, B.P. Patil and D.K. Gautam, "Performance Evaluation of Squaring Operation by Vedic Mathematics", IETE Journal of Research, vol.57, Issue 1, Jan-Feb 2011.

[15] Aniruddha Kanhe, Shishir Kumar Das and Ankit Kumar Singh, "Design and Implementation of Low Power Multiplier Using Vedic Multiplication Technique”, (IJCSC) International Journal of Computer Science and Communication Vol. 3, No. 1, January-June 2012, pp. 131-132

[16] Umesh Akare, T.V. More and R.S. Lonkar, "Performance Evaluation and Synthesis of Vedic Multiplier", National Conference on Innovative Paradigms in Engineering \& Technology (NCIPET2012), proceedings published by International Journal of Computer Applications (IJCA), 2012.

\section{AUTHOR'S PROFILE}

Pushpalata Verma obtained her B. Sc (Computer Science) in 2005 from B.M.M., Bhilai and M. Sc. (Computer Science) in 2007 from S.S.M.V., Bhilai. She is pursuing her M.E. in Computer Technology and Application (CTA) from Shri Shankaracharya College of Engineering \& Technology (SSCET) Bhilai, Chhattisgarh. Presently she is working as Assistant Professor in Central College of Engineering \& Technology (CCEM), Raipur, Chhattisgarh, India. 